\title{
Acopladores à Fibra Óptica Monomodo- Multimodo como Dispositivos para Telecomunicações e Sensores
}

\author{
Ricardo M. Ribeiro, Yanes C. Balod e Andrés P. L. Barbero
}

\begin{abstract}
Resumo - Este trabalho mostra pela primeira vez a resposta espectral (banda-C) de acopladores $2 \times 2$ nãorecíprocos formados pela fusão de fibra óptica monomodo com multimodo. O dispositivo obtido pode ser classificado como do tipo MMI (multimode-interference) que dependendo das condições de injeção de luz e das portas de entrada/saída utilizadas, se comporta como um de três diferentes tipos de filtros ópticos ou interferômetro multimodo. Algumas utilidades do dispositivo são também descritas neste trabalho.
\end{abstract}

Palavras-chave - Acoplador, filtro óptico, interferência multimodo, não-reciprocidade, dispositivo a fibra óptica.

Abstract - This paper show for the first time the spectral response (C-band) of $2 \times 2$ non-reciprocal fibrecoupler built from single-mode fused with multi-mode fibre. Such multimode interference (MMI) device may exhibit different spectral transmissions as an optical filter or interferometer depending on the detailed launch conditions. Some applications are also described.

Keywords - Coupler, optical filter, multimode interference, non-reciprocity, fibre optic device.

\section{INTRODUÇÃO}

Acopladores SM/MM formados pela fusão ou contato tangencial entre fibras ópticas monomodo (SM) com multimodo (MM) não são usuais. Porém, foram descritos na literatura em 1985-1987 [1-3] como sendo dispositivos de natureza não-recíproca. Algumas aplicações interessantes foram já na época realizadas ou propostas como, por exemplo, para compor uma rede óptica em barramento, construir linhas de atraso por anéis de recirculação em fibras, OTDR e em sensores. Desde então, não foi possível encontrar mais qualquer registro de trabalhos publicados que tratem dos tais acopladores $\mathrm{SM} / \mathrm{MM}$.

Por um outro lado, em 1973 foi mostrado [4] que o fenômeno do re-imageamento óptico (self-imaging) pode ocorrer em guias de ondas multimodo de índice degrau (SI) [5]. O fenômeno do re-imageamento óptico é mais conhecido como aquele que ocorre em fibras ópticas GIMM de Telecomunicações, onde sob o ponto de vista da

Ricardo M. Ribeiro, rmr@pq.cnpq.br, Yanes C. Balod, yanes@pobox.com, Andrés P. L. Barbero, Pablo@telecom.uff.br, Laboratório de Comunicações Ópticas, Departamento de Engenharia de Telecomunicações, Universidade Federal Fluminense, 24.210-240 Niterói RJ Brasil. Este trabalho foi parcialmente financiado pelo $\mathrm{MCT} / \mathrm{CNPq}$ e Faperj.
Óptica Geométrica (raios) a propagação ocorre num padrão senoidal com periodicidade (pitch) típica de $\sim 1 \mathrm{~mm}$, como se fosse um efeito lente de focalização e desfocalização da luz dentro da fibra [6]. Dispositivos baseados no efeito de re-imageamento em guias ou fibras ópticas multimodo são genericamente chamados de dispositivos MMI. Até aproximadamente 1995 [5] as investigações de dispositivos MMI se concentraram em guias ópticas. Desde então, pode-se observar uma quantidade razoável de trabalhos publicados sobre investigações teóricas e experimentais de dispositivos MMI baseados em fibras ópticas, principalmente nos últimos 6 anos [6-15], basicamente consistindo de um segmento de fibra multimodo [14] ou de fibra fotônica [15] emendado por fusão numa fibra óptica monomodo.

No melhor do conhecimento dos autores, este trabalho mostra pela primeira vez resultados de investigações experimentais de acopladores SM/MM também operando como dispositivos MMI. Mais especificamente, três são as novidades aqui relatadas: A resposta espectral propriamente dita (na banda-C) do acoplador SM/MM sob dois diferentes regimes de propagação e nas diversas combinações de portas de entrada/saída. Como conseqüência, também é aqui demonstrado que o dispositivo pode ter múltiplas utilidades, como exibir comportamento de filtro espectral de várias modalidades ou de interferômetro multimodal. A terceira contribuição, refere-se a descrição de três aplicações específicas utilizando o dispositivo, mas não ainda efetivamente realizadas.

\section{CONFIGURAÇÃO EXPERIMENTAL}

A Figura 1 mostra esquematicamente a configuração experimental utilizada para caracterizar o acoplador $\mathrm{SM} / \mathrm{MM}$ no domínio espectral. O acoplador SM/MM foi construído utilizando a técnica convencional de fusão e estiramento de fibra óptica monomodo SI padrão G652 $(9 / 125 \mu \mathrm{m})$ com outra multimodo GI padrão G651 $(62,5 / 125 \mu \mathrm{m})$ enroscada uma com a outra de forma a ficar com $\sim 10 \mathrm{~mm}$ de comprimento de junção. A fabricação foi ajustada de forma que 2-5\% da luz injetada em $1550 \mathrm{~nm}$ pudesse sair pela fibra SM quando a injeção se desse pela fibra MM. Um laser sintonizável nos canais DWDM ao longo da banda-C (1527-1563 nm) foi usado para interrogar opticamente o dispositivo. O laser acoplado num pigtail de fibra SM passa por um isolador a fibra antes de ser injetado no acoplador SM/MM em teste. 


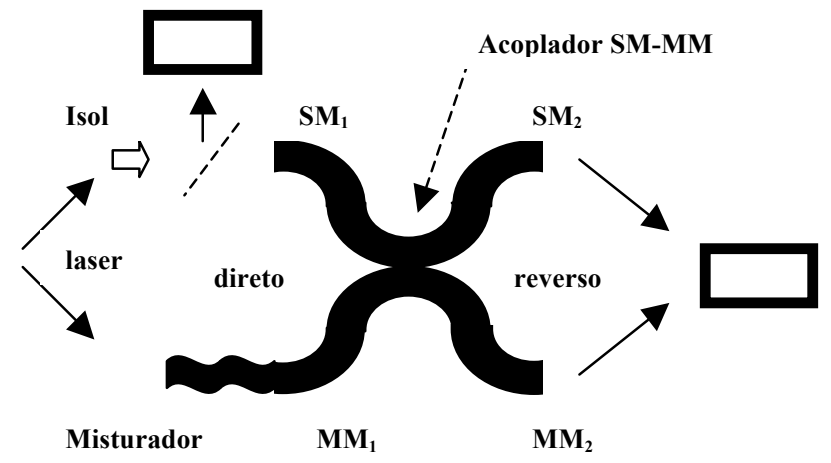

Fig. 1. Configuração experimental para a caracterização espectral do dispositivo MMI consistindo de um acoplador SM/MM. Os retângulos simbolizam medidores de potência óptica.

Com o uso de um medidor de potência óptica, fez-se primeiramente uma medida da potência do laser em função do comprimento de onda para servir como espectro de referência. Foi obtida uma resposta praticamente plana. A seguir, o acoplador SM/MM foi caracterizado no domínio espectral. A luz foi injetada na fibra SM ou MM. Nesta última, a injeção foi feita diretamente do pigtail em fibra SM do laser - modos de baixa ordem - ou através de um misturador de modos - modos distribuídos equilibradamente. As medidas foram primeiramente feitas no sentido direto e depois repetidas no sentido reverso, conforme ilustrado na Figura 1. Naturalmente que a atribuição de sentido "direto" ou "reverso" foi inteiramente arbitrária. Desta forma, foi obtido um total 12 gráficos espectrais, mas nem todos estes foram necessários mostrar neste trabalho. A resposta espectral do misturador de modos (mode-scrambler) foi também previamente medida como sendo absolutamente plana na banda-C.

A Figura 2 mostra uma fotografia do aparato experimental utilizado para a caracterização do dispositivo. O laser sintonizável não é mostrado e o acoplador SM/MM aparece na parte superior da fotografia sobre um pequeno retângulo de papel branco. Para a injeção e medida da potência dos sinais ópticos, pigtails de fibras SM e MM foram emendadas por fusão nas respectivas fibras de entrada/saída do dispositivo.

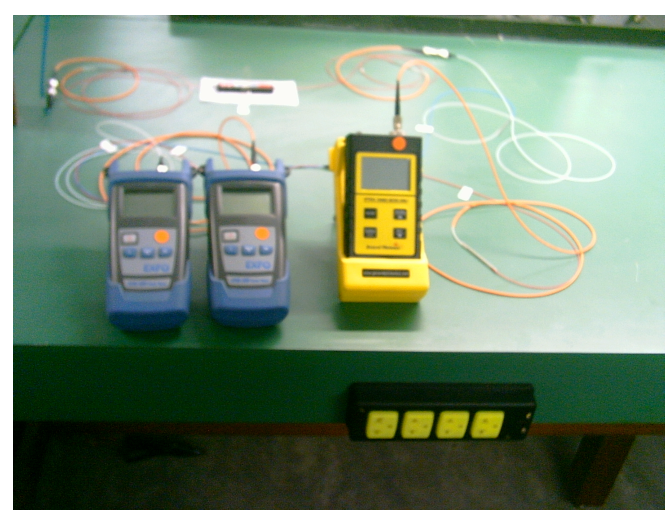

Fig. 2. Fotografia da configuração experimental da Figura 1. Pode-se notar o acoplador SM/MM (com pigtails em terminação FC) na parte superior da figura sobre um retângulo de papel branco.

\section{RESULTADOS E DISCUSSÕES}

As Figuras 3 e 4 mostram a resposta espectral da perda por inserção e acoplamento cruzado, respectivamente, quando a luz era injetada em cada uma das portas SM, ou seja, na $\mathrm{SM}_{1}$ (sentido direto) e depois na $\mathrm{SM}_{2}$ (sentido reverso). As Figuras 3 referem-se a saída pelas portas SM, enquanto que as Figuras 4 pelas portas MM.

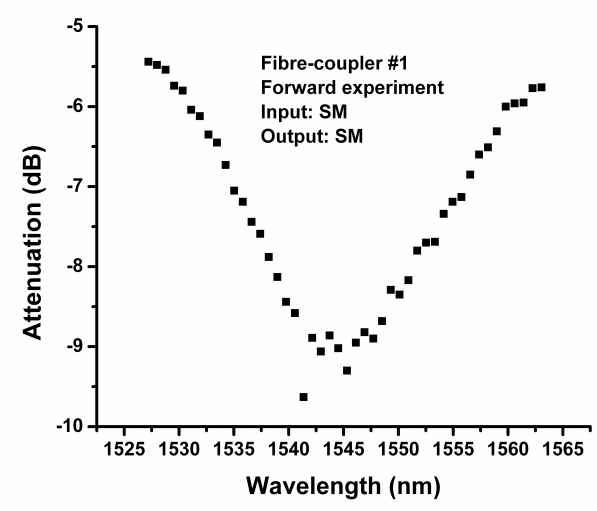

3(a)

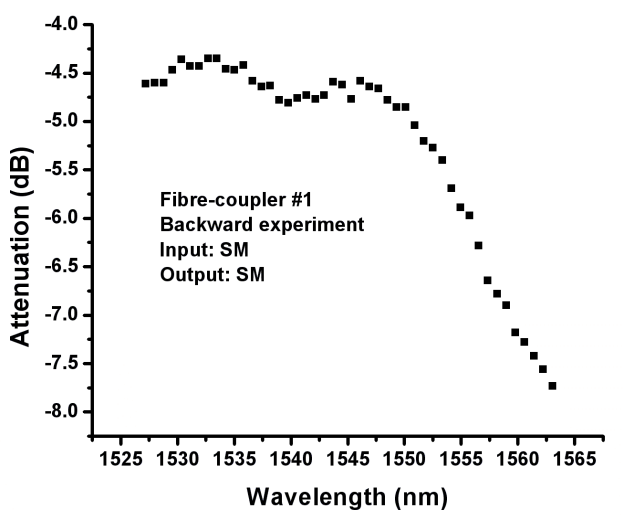

3(b)

Fig. 3. Resposta espectral da perda por inserção no (a) sentido direto $\mathrm{SM}_{1} \rightarrow \mathrm{SM}_{2}$ e no (b) sentido reverso $\mathrm{SM}_{2} \rightarrow \mathrm{SM}_{1}$

Comparando a Figura 3a com a 3b, observa-se claramente não-reciprocidade quando a foto-detecção acontece pela mesma fibra onde a luz foi originalmente injetada. Trata-se de não-reciprocidade na perda por inserção. O espectro transmitido por cada fibra SM não é o mesmo, pois as defasagens relativas das componentes (modos) interferentes não são as mesmas, ou seja, dependem da injeção de luz ser feita no sentido direto ou reverso. A estrutura de guia de onda MM (junção do acoplador) experimentada pelo sinal injetado a partir das fibras SM não é o mesma. Acredita-se então que esta seja a origem da não-reciprocidade que se verifica na perda por inserção do acoplador SM/MM. 
Comparando a Figura 4a com a 4b, também claramente observa-se não-reciprocidade quando a foto-detecção acontece pela fibra MM que não é onde a luz foi originalmente injetada. Neste caso, a não-reciprocidade observada acontece na perda por acoplamento cruzado, e não por inserção como no caso anterior. A origem da nãoreciprocidade no acoplamento cruzado deve-se ao mecanismo de não-reciprocidade da perda por inserção mencionado anteriormente, em combinação com a complementaridade dos espectros de saída devido a conservação de energia, conforme explicação adicional no parágrafo seguinte.

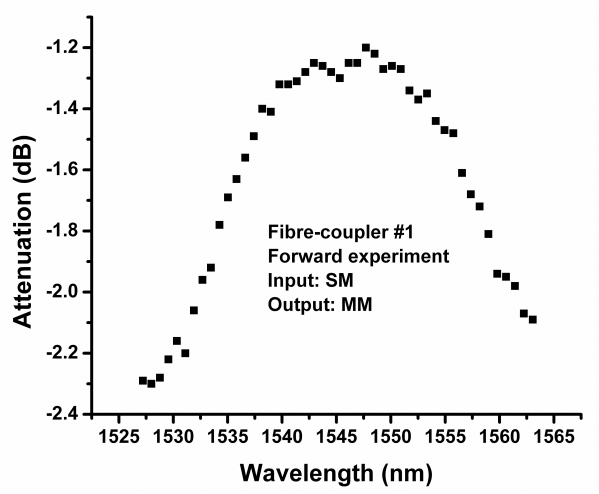

4(a)

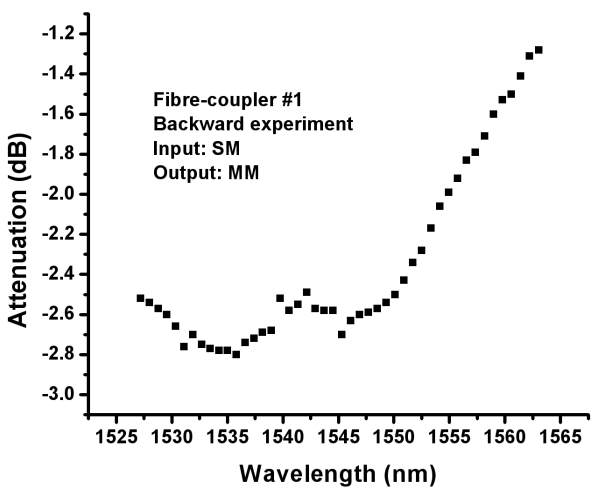

4(b)

Fig. 4. Resposta espectral da perda por acoplamento cruzado no (a) sentido direto $\mathrm{SM}_{1} \rightarrow \mathrm{MM}_{2}$ e no (b) sentido reverso $\mathrm{SM}_{2} \rightarrow \mathrm{MM}_{1}$.

Comparando as Figuras 3a com 4a, assim como 3 b com $4 \mathrm{~b}$, observa-se que os gráficos possuem entre si um caráter complementar em seu formato. Isto está compatível com conservação da energia, onde praticamente toda a luz injetada no dispositivo é compartilhada na saída pelas duas portas SM e MM. Naturalmente que existem perdas adicionais por irradiação para o espaço livre e por diretividade (retro-espalhamento).

A Figura 3a mostra que no sentido direto $\mathrm{SM}_{1} \rightarrow \mathrm{SM}_{2}$, o dispositivo se comporta como um filtro rejeita-banda (notch-filter) com profundidade de $\sim 4 \mathrm{~dB}$ em torno de $1545 \mathrm{~nm}$ e largura espectral $(-3 \mathrm{~dB})$ de $\sim 29 \mathrm{~nm}$, que o torna potencialmente útil para aplicações CWDM.
A Figura $3 \mathrm{~b}$ mostra que no sentido reverso $\mathrm{SM}_{2} \rightarrow \mathrm{SM}_{1}$, o dispositivo se comporta como um filtro tipo borda (edgefilter) apresentando transmissão razoavelmente plana na faixa $1525-1550 \mathrm{~nm}$ e a seguir o filtro de borda propriamente dito com $+0,23 \mathrm{~dB} / \mathrm{nm}$ de inclinação (rolloff).

A Figura 4a mostra que no sentido direto $\mathrm{SM}_{1} \rightarrow \mathrm{MM}_{2}$, o dispositivo se comporta como um filtro passa-banda (band-pass) em torno de $1545 \mathrm{~nm}$, embora seja de baixa amplitude de transmissão $(\sim 1 \mathrm{~dB})$.

A Figura $4 \mathrm{~b}$ mostra que na direção reversa $\mathrm{SM}_{2} \rightarrow \mathrm{MM}_{1}$ o dispositivo se comporta como um filtro tipo borda (edgefilter), porém agora com inclinação oposta a da Figura 3b, apresentando transmissão razoavelmente plana de 1525$1550 \mathrm{~nm}$ e a seguir o filtro de borda propriamente dito com - 0,10 dB/nm de inclinação.

Pode-se notar das Figuras 3 e 4, que o comportamento passa-banda/rejeita-banda ou filtro de borda não aparece simplesmente em função do intervalo espectral considerado [14]. Basta notar que as regiões espectralmente planas do filtro de borda não correspondem, quando se compara com o dispositivo funcionando como filtro passa-banda/rejeita-banda.

Todas as Figuras 5-8 referem-se a injeção de luz através das portas $\mathrm{MM}$ e dois diferentes regimes de modos injetados nas mesmas. O primeiro regime refere-se a injeção de modos com distribuição equilibrada, para isto usando um misturador de modos (mode-scrambler) conforme mostrado na Figura 1. Este primeiro regime corresponde a uma "interferência multimodal geral" [5]. O segundo regime compreende a injeção de luz na fibra MM através da fibra SM, obtendo-se então modos de baixaordem, também ilustrado na Figura 1. Neste último caso, espera-se que a maior parte da energia seja acoplada no modo $\mathrm{LP}_{01}$ propagante pela fibra MM. Supondo uma conexão perfeitamente concêntrica entre as fibras, apenas os modos simétricos $\mathrm{LP}_{0 \mathrm{~m}}$ serão gerados. Este segundo regime corresponde a uma "interferência multimodal restrita" [5].

Conforme mostrado na Figura 5, quando modos de baixa-ordem são injetados, observa-se um espectro de saída consistindo de rápidas oscilações com a variação do comprimento de onda com intervalos entre vales $<10 \mathrm{~nm}$ e amplitude de profundidade na faixa 6-12 dB. Em qualquer comprimento de onda considerado, o sinal é bem atenuado em comparação com o injetado. Por exemplo em $\sim 1538 \mathrm{~nm}$ temos $12 \mathrm{~dB}$ de atenuação, o menor valor.

O comportamento mostrado na Figura 5 é similar ao do fenômeno do re-imageamento [5] característico de estruturas SMS quando o comprimento físico da guia multimodo é alterado. Desta forma, o presente dispositivo poderá ser útil como um sensor de tensão mecânica $[5,10]$ (strain-sensor).

A Figura 5 mostra então o espectro de saída quando modos de baixa-ordem são injetados no sentido direto $\mathrm{MM}_{1} \rightarrow \mathrm{SM}_{2}$. Um resultado similar foi obtido (mas não é aqui mostrado), no sentido reverso, porém apresentando um ligeiro deslocamento de fase. 


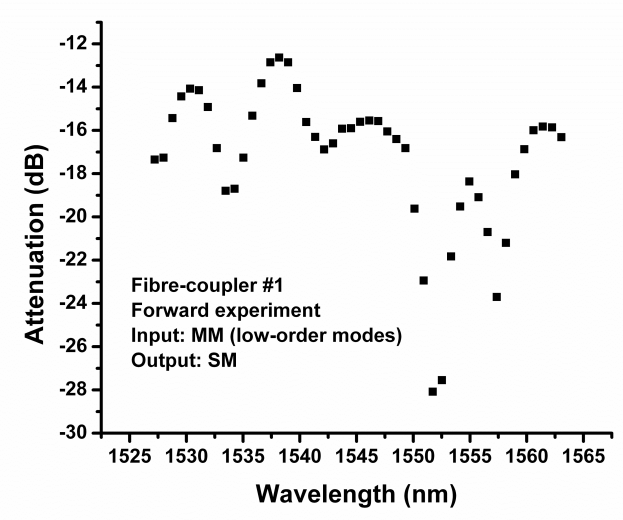

Fig. 5. Resposta espectral da perda por acoplamento cruzado no sentido direto $\mathrm{MM}_{1} \rightarrow \mathrm{SM}_{2}$ quando modos de baixa ordem são injetados.

A Figura 6 mostra o espectro de saída quando modos de baixa-ordem são injetados no sentido direto $\mathrm{MM}_{1} \rightarrow \mathrm{MM}_{2}$. Novamente, um resultado similar (não é aqui mostrado) foi obtido para o sentido reverso. Fazendo uma comparação entre as Figuras 5 e 6 , observa-se similaridades entre os picos e vales, porém com uma profundidade de modulação bastante reduzida quando o sinal óptico sai do dispositivo pelas portas MM.

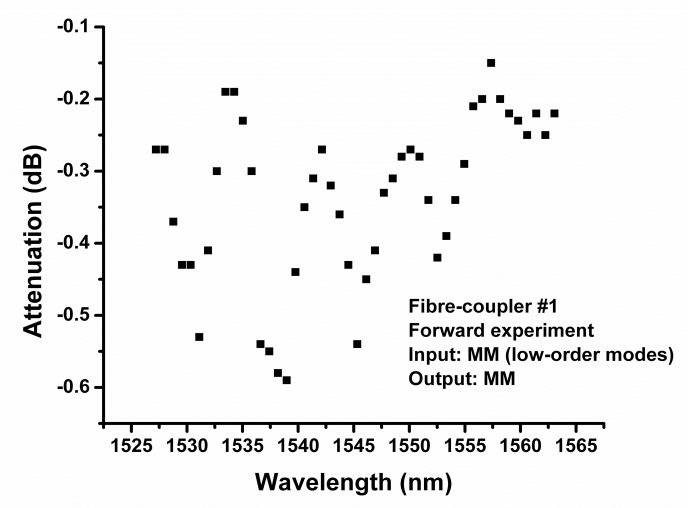

Fig. 6. Resposta espectral da perda por inserção no sentido direto $\mathrm{MM}_{1} \rightarrow \mathrm{MM}_{2}$ quando modos de baixa ordem são injetados.

As Figuras 3 e 4 mostram respostas espectrais similares aos de acopladores de fibras SM $2 \times 2$ convencionais ou do tipo WDM, porém apresentam não-reciprocidade e diferenças quando se trata de comportamento tipo filtro de borda. Entretanto as Figuras 5 e 6 mostram um comportamento bem diferente da resposta espectral de acopladores MM 2x2 convencionais.

As Figuras 7 e 8 mostram o espectro de saída pelas portas $\mathrm{SM}_{1}$ e $\mathrm{MM}_{1}$, respectivamente, quando modos com distribuição equilibrada são injetados no sentido reverso através da porta $\mathrm{MM}_{2}$. As Figuras 7 e 8 mostram que vales e picos já não são bem definidos, tal como se tem observado em dispositivos SMS [7]. De fato, no decorrer dos experimentos, observou-se a saída de sinais ópticos instáveis frente às diversas perturbações externas como vibrações, contato com a fibra, etc [15-17]. Tais instabilidades são características de interferômetros a fibra multimodo [6,8,15-17].

Quando o sinal era injetado pelas portas SM, o dispositivo podia operar como uma das três modalidades de filtro espectral qualquer que fosse a porta de saída. Quando modos de baixa-ordem eram injetados no sentido $\mathrm{MM} \rightarrow \mathrm{SM}$, o dispositivo era estável frente a perturbações externas e apresentava espectro similar com vários mínimos de atenuação. Porém (ver Figuras 6 e 8), quando a saída acontecia pelas portas-MM, o dispositivo se tornava instável. Nesta última configuração, o dispositivo simultaneamente acoplava luz e gerava interferência multimodo. Isto pode ser comparado aos sensores tipo modalmétrico $[6,8,15-17]$ baseados na combinação de um acoplador a fibra óptica SM convencional com uma emenda por fusão tipo SM/MM. A Figura 7 também mostra um sinal óptico de saída instável.

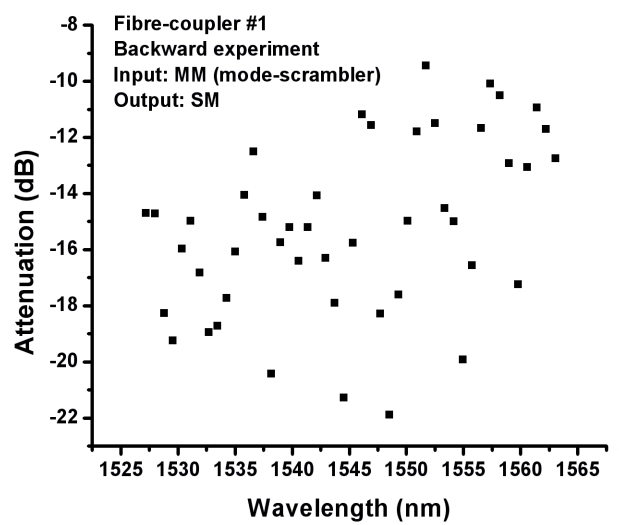

Fig. 7. Resposta espectral da perda por acoplamento cruzado no sentido reverso $\mathrm{MM}_{2} \rightarrow \mathrm{SM}_{1}$ quando modos distribuídos de forma equilibrada são injetados.

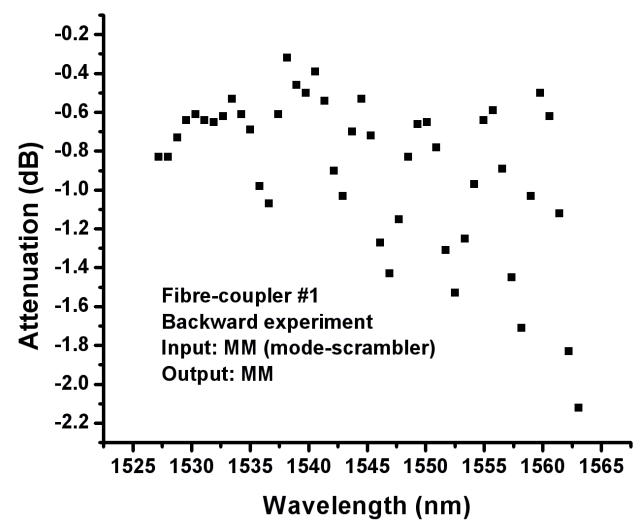

Fig. 8. Resposta espectral da perda por inserção no sentido reverso $\mathrm{MM}_{2} \rightarrow \mathrm{MM}_{1}$ quando modos distribuídos de forma equilibrada são injetados. 


\section{ANÁLISE DO DISPOSITIVO - MEDIDOR DE COMPRIMENTO DE ONDA}

Injetando luz na porta $\mathrm{SM}_{2}$, as saídas por $\mathrm{SM}_{1}$ (Figura 3b) e $\mathrm{MM}_{1}$ (Figura $4 \mathrm{~b}$ ) consistem em respostas tipo filtro de borda, porém com inclinações opostas. Exceto pelo fato de que a perda é desigual na saída de cada braço, o dispositivo aqui tratado poderia constituir-se como um monitor auto-normalizado de comprimento de onda numa só unidade [14]. Para isto, dois medidores de potência óptica são utilizados, um para $\mathrm{SM}_{1}$ e outro para $\mathrm{MM}_{1}$. Os resultados das medidas são por fim subtraídos um do outro, o que pode fornecer (após calibração) um valor bastante preciso de $\lambda[14]$.

\section{ANÁLISE DO DISPOSITIVO - REDE EM BARRAMENTO}

Analisando o dispositivo funcionando nos sentidos diretos $\mathrm{MM}_{1} \rightarrow \mathrm{SM}_{2}$ (Figura 5) e $\mathrm{MM}_{1} \rightarrow \mathrm{MM}_{2}$ (Figura 6), pode-se notar que de fato possui o comportamento requerido para compor o nó de uma rede em barramento através de uma fibra MM, enquanto as fibras SM fazem a derivação (tap) da rede. Em toda a banda $\mathrm{C}$, de fato quase toda a potência injetada prossegue pela fibra MM (ver Figura 6), enquanto que apenas uma fração é derivada para a fibra SM (ver Figura 5). Entretanto, conforme mostra a Figura 5, ao longo da banda $\mathrm{C}$ a resposta espectral (atenuação) é bastante não-uniforme. Por exemplo, o canal em $1537,5 \mathrm{~nm}$ é atenuado de $\sim 13 \mathrm{~dB}$ enquanto que 1552 $\mathrm{nm}$ é atenuado de $\sim 28 \mathrm{~dB}$. Esta falta de equalização terá de ser levada em conta num projeto de rede em barramento com multiplexação em comprimento de onda.

As Figuras 7 e 8 no sentido reverso, porém com modos injetados com distribuição equilibrada, mostram mais uma vez que a maior parte da potência é mantida na fibra MM, enquanto que uma pequena fração é desviada para a fibra SM. Porém agora, vales e picos não ficam definidos pois o dispositivo passa a ser instável devido as acentuadas interferências multimodais.

\section{ANÁLISE DO DISPOSITIVO - ANEL DE RECIRCULAÇÃO}

Uma forma de se construir um anel de re-circulação é injetar a luz pela fibra SM e emendar por fusão os dois braços MM do acoplador para formar o anel [3]. As Figuras 4 (SM $\rightarrow \mathrm{MM})$ mostram que a fração de luz injetada no anel depende do comprimento de onda e do sentido da propagação no dispositivo, que não é recíproco. Por exemplo, o canal em $1545 \mathrm{~nm}$ é atenuado de $\sim 1,3 \mathrm{~dB}$ no sentido direto e $2,6 \mathrm{~dB}$ no sentido reverso. No sentido direto, enquanto $1545 \mathrm{~nm}$ é atenuado de 1,3 dB, 1527,5 nm é atenuado de 2,3 dB.

Após o sinal óptico completar uma volta no anel de fibra MM, passa pelo acoplador com injeção de modos de baixa ordem se o acoplamento modal puder ser desprezado. Neste caso, é de se esperar um comportamento estável do dispositivo e as Figuras $5(\mathrm{MM} \rightarrow \mathrm{MM})$ e $6(\mathrm{MM} \rightarrow \mathrm{MM})$ mostram que de fato a maior parte da luz permanece no anel enquanto que uma fração prossegue pela fibra SM. Entretanto, tudo isto mais uma vez depende do comprimento de onda, porém com uma dependência completamente diferente daquela mostrada na injeção no anel (ver Figuras 4).

\section{CONCLUSÕES}

É aqui pela primeira vez descrito a caracterização espectral na banda $\mathrm{C}$ de um acoplador não-recíproco a fibra SM/MM. Os resultados de nossos experimentos mostraram que o acoplador exibe resposta de filtro (rejeitabanda, passa-banda ou de borda) ou interferômetro multimodo (MMI) não-recíproco, ou seja, dependente das portas de entrada e saída, assim como das condições de injeção de modos.

Três exemplos de aplicações foram descritos com o objetivo de mostrar o potencial do dispositivo quando se considera o tráfego de sinais na banda-C.

O presente dispositivo pode ser compacto e útil para aplicações em Telecomunicações e Sensores baseados em fibras ópticas. Entretanto, ainda requer o desenvolvimento de um modelo teórico preciso de forma a projetá-lo e otimizá-lo para alguma aplicação específica.

\section{REFERÊNCIAS}

[1] M. S. Whalen and T. H. Wood, "Effectivelly nonreciprocal evanescent-wave optical-fibre directional coupler", Electronics Letters, 21, 5, pp. 175-176, 1985.

[2] H. C. Chang, H. S. Huang and J. Wu, "Wave Coupling Between Parallel Single-Mode and Multimode Optical Fibers", IEEE Transactions on Microwave Theory and Techniques, MTT-34, 12, pp. 1337-1343, 1986 .

[3] R. J. Boughton, M. Farhadiroushan, D. Kreit, R. C. Youngquist and I. P. Giles, "Optical-fiber systems with nonreciprocal couplers”, Optics Letters, 12, 3, pp. 205-207, 1987.

[4] O. Bryngdahl, "Image formation using self-imaging techniques", Journal of Optical Society of America, 63, 4, pp. 416-419, 1973.

[5] L. B. Soldano and E. C. M. Pennings, "Optical Multi-Mode Interference Devices Based on Self-Imaging: Principles and Applications", Journal of Lightwave Technology, 13, 4, pp. 615627, 1995.

[6] R.M. Ribeiro and M.M. Werneck, "An intrinsic graded-index multimode optical fibre strain-gauge" Sensors and Actuators A, 111, pp. 210-215, 2004.

[7] A. Kumar, R. K. Varshney, S. Antony snd P. Sharma, "Transmission characteristics of SMS fiber optic sensor structures", Optics Communications, 219, pp. 215-219, 2003.

[8] W. S. Mohammed, A. Mehta and E. G. Johnson, "Wavelength tunable fiber lens based on multimode interference", Journal of Lightwave Technology, 22, 2, pp. 469-477, 2004.

[9] G. A. Sanchez, A. M. Rios, D. A. M. Arrioja, I. T. Gomez, R. S. Aguilar and J. A. Chavez, "Enhanced tuning mechanism in fibre laser based on multimode interference effects", Electronics Letters, 42, 23, pp. 1337-1338, 2006.

[10] E. Li, "Temperature compensation of multimode-interferencebased fiber devices", Optics Letters, 32, 14, pp. 2064-2066, 2007.

[11] B. M. Shalaby, V. Kermene, D. Pagnoux and A. Barthelemy, "Transverse mode control by self-imaging process in a multiple fibre laser using a single-mode feedback loop", Journal of Optics A: Pure and Applied Optics, 10, pp. 115303, 2008. 
[12] W. Shin, K. Oh, B. A. Yu, Y. L. Lee, Y. C. Noh, J. Lee and D. K. Ko, "All-fiber wavelength tunable and mode convertible bandpass filter for optical inter-connections", OFC/NFOEC, paper OWI1, 2008.

[13] Q. Wang, G. Farrel and W. Yan, "Investigation on single-modemultimode-single-mode fiber structure", Journal of Lightwave Technology, 26, 5, pp. 512-519, 2008.

[14] A. M. Hatta, G. Farrell, Q. Wang, G. Rajan, P. Wang and Y. Semenova, "Ratiometric wavelength monitor based on singlemode-multimode-singlemode fiber structure, Microwave and Optical Technology Letters, 50, 12, pp. 3036-3039, 2008.

[15] Y. Wang, H. Cai, R. Qu, Z. Fang, E. Marin and J. P. Meunier, "Specklegram in a grapefruit fiber and its response to external mechanical disturbance in a single-multiple-single mode fiber structure", Applied Optics, 47, 20, pp. 3543-3548, 2008.

[16] I. Oanca, G.Y. Yang, J. Katsifolis and E. Tapanes, "Simultaneous wavelength multiplexed fiber optic communications and cable integrity monitoring technique", CLEO, paper WP4, pp. 106-107, $16^{\text {th }}$ July, 1997.

[17] R. M. Ribeiro, Y. C. Balod, A. P. L. Barbero, M. B. de Carvalho e P. A. M. dos Santos, "Monitoramento da Integridade Física de Cabos Ópticos de Telecomunicações Usando um Sistema Modalmétrico no Domínio do Tempo", artigo 42633, XXVI Simpósio Brasileiro de Telecomunicações, SBrT'08, 02-05 de Setembro, Rio de Janeiro, RJ, 2008. 